\title{
TRAUMATISMOS CAUSADOS POR EL TRÁNSITO EN EL PERÚ. ¿DÓNDE ESTAMOS Y HACIA DÓNDE VAMOS?
}

\author{
[ROAD TRAFFIC INJURIES IN PERU. WHERE ARE WE AND WHAT NEXT?]
}

\author{
J. Jaime Miranda ${ }^{1,2,3, a}$, Luis Huicho $2,4,5, b$
}

El reconocimiento de los traumatismos causados por el tránsito como un problema de salud pública viene tomando fuerza en los últimos años ${ }^{(1,2)}$. La Revista Peruana de Medicina Experimental y Salud Pública no es ajena a este progreso y dedica este número especial de la Revista a dicho tema. Incluso la terminología utilizada comúnmente, "accidentes de tránsito", es una barrera para lograr comprender las distintas aristas de este problema. En contraste, el término "traumatismos causados por el tránsito" fue ampliamente adoptado en el Informe mundial sobre prevención de los traumatismos causados por el tránsito, elaborado por la Organización Mundial de la Salud en el año $2004{ }^{(1)}$. El uso, para algunos inadecuado, del término accidentes de tránsito, limita la comprensión de lo mucho que se puede hacer para prevenir estos eventos. Dado el uso cotidiano del término "accidentes de tránsito" en nuestro medio, y a fin de generar familiaridad y contribuir en el uso adecuado del término "traumatismos causados por el tránsito", en este artículo se usará, en la medida de lo posible, este último. Este editorial no intenta reflejar una revisión de lo publicado sobre el tema en nuestro medio; en cierta medida este número especial, a través de sus distintas contribuciones, reflejará la evidencia disponible sobre el tema en el país.

\section{EL CONTEXTO DE LOS TRAUMATISMOS CAUSADOS POR EL TRÁNSITO EN EL PERÚ}

Es igualmente importante conocer cuáles son los actores e interlocutores claves involucrados en la toma de medidas para minimizar este problema. No es sino hasta hace poco que el sector salud se viene involucrando en este asunto; tradicionalmente, su participación ha estado circunscrita a acciones recuperativas en las víctimas de un evento vinculado con el tránsito. Sin embargo, los retos en este campo, como se indica en este editorial, son mayores.

A diferencia de muchas prioridades de salud pública para nuestro país, el sector salud no ha demostrado un liderazgo en el tema de traumatismos causados por el tránsito. Resulta sencillo reconocer y afirmar que las agendas de tuberculosis, VIH/Sida, malaria, desnutrición infantil, entre otros, son largamente dominadas por el sector salud. No ocurre lo mismo con la agenda de los traumatismos causados por el tránsito. En este ámbito participan, en algún grado, pero con mayor preponderancia que el sector salud, los medios de comunicación, el sector transporte, la policía y las empresas aseguradoras privadas. Una dificultad adicional es que cada una de estas entidades enfoca el tema desde su propia perspectiva: la noticia, el vehículo, el evento, el siniestro. El sector salud contribuye con una perspectiva adicional: la salud de los afectados y cómo se pueden prevenir los eventos. En medio de este contexto con múltiples actores, es claro que no hay un mensaje único y si lo hubiera, el liderazgo no recae en el sector salud. Este contexto no es necesariamente malo, pero plantea un reto -y quizás la oportunidad- de poder tener una acción de respuesta concertada multisectorial y multidisciplinaria.

De esta variedad de actores, resulta satisfactorio contar en este número especial con contribuciones de diversos grupos locales ${ }^{(3-8)}$ incluyendo el Ministerio de Salud ${ }^{(9)}$, el Programa de Investigación en Accidentes de Tránsito ${ }^{(10,11)}$ y la Defensoría del Pueblo del Perú ${ }^{(12)}$. Debemos resaltar que, como parte del proceso de consolidación de la Revista Peruana de Medicina Experimental y Salud Pública, se cuente en este número con la contribución de grupos académicos de investigación internacionales con aportaciones procedentes de la Universidad de Johns Hopkins y la Universidad de Washington, ambas de los Estados Unidos ${ }^{(13,14)}$.

\footnotetext{
Programa de Investigación en Accidentes de Tránsito, Salud Sin Límites Perú. Lima, Perú.

Facultad de Medicina "Alberto Hurtado", Universidad Peruana Cayetano Heredia. Lima, Perú

CRONICAS, Centro de Excelencia en Enfermedades Crónicas, Universidad Peruana Cayetano Heredia. Lima, Perú

Departamento de Pediatría, Instituto Nacional de Salud del Niño. Lima, Perú.

Facultad de Medicina, Universidad Nacional Mayor de San Marcos. Lima, Perú

Médico, Magíster y Doctor en Epidemiología; ${ }^{b}$ Médico Pediatra, Doctor en Medicina.
} 
Vale la pena expresar que, a pesar de que en este número no se ha podido asegurar la participación de entidades con otros puntos de vista complementarios y relevantes, como por ejemplo del Ministerio de Transportes y Comunicaciones, las páginas de esta tribuna se mantienen abiertas para eventuales contribuciones de todo aquel interesado en este tema.

\section{UNA PERSPECTIVA MACRO DE LOS TRAUMATISMOS CAUSADOS POR EL TRÁNSITO EN EL PERÚ}

Los traumatismos causados por el tránsito tienen determinantes múltiples $\mathrm{y}$, por tanto, no pueden ser enfrentados solamente desde una perspectiva de salud. De hecho, el último informe elaborado por la Organización Mundial de la Salud sobre el tema, que incluye información sobre el Perú (2), sienta las bases para establecer pautas de monitoreo de progresos en temas de seguridad vial y, por ende, un monitoreo de su potencial impacto y progresos en términos de salud pública. El informe es analizado en este número especial, por un grupo de expertos internacionales, vinculando el tema de traumatismos causados por el tránsito a un tema de desarrollo ${ }^{(13)}$.

Sin duda la epidemiología y la salud pública tienen la capacidad de incorporar una perspectiva más sistemática al problema, enfatizando la necesidad de contar con información adecuada sobre este, que permita definir su naturaleza, su magnitud, su distribución, los factores de riesgo y las medidas de prevención que por consiguiente se pueden desarrollar. Pueden permitir, igualmente, la incorporación de la evidencia científica en la consideración de las intervenciones que se diseñen e implementen, para aumentar sus probabilidades de éxito, así como la inclusión de un componente de monitoreo y evaluación con indicadores claramente definidos para medir su impacto.

Se requiere, sin embargo, de una aproximación más amplia, a fin de poder asegurar las expectativas sobre las reales probabilidades de éxito de las estrategias de prevención y atenuación del problema. Para ello es necesario que se considere el análisis del contexto nacional y local, sus tendencias actuales y futuras y de qué manera las estrategias de prevención y atenuación se insertan en el contexto más amplio.

El análisis del problema debe por fuerza considerar las tendencias de la globalización en nuestro país. El proceso de urbanización a nivel nacional y en las diversas regiones del país va a continuar, a diferente velocidad y con diferentes grados de planificación, al igual que el proceso de avance de la red vial nacional y la red vial dentro de cada ciudad. Las limitaciones en concebir e impulsar un proceso de desarrollo urbano planificado, que considere el bienestar de las personas antes que la rentabilidad de las inversiones, establece nuevos retos para la salud pública. Si las ciudades y sus vías de comunicación se multiplican caóticamente, como ocurre en la actualidad, los factores de riesgo para la ocurrencia de accidentes continuarán aumentando exponencialmente. Este es un problema sobre el que debe abrirse un debate amplio y sostenido que incluya a la sociedad civil, pues no habrá iniciativa que pueda tener éxito sin ciudadanos conscientes de sus derechos y sus deberes.

El proceso de migración de las áreas rurales a las ciudades y de las ciudades del interior del país a las grandes ciudades incluida la capital, que es cada vez más predominante, ligado al proceso de globalización más amplio, supone también un reto adicional. Esto es palpable en la medida que quienes migran tienen sus propios patrones culturales o se ven forzados a adaptar patrones de conducta que no sintonizan necesariamente con las normas, lo que se traduce en patrones de adaptación que pueden constituir obstáculos para el logro de los objetivos de las estrategias de prevención y seguridad vial.

Un ejemplo de estos retos, importante para la conceptualización del problema y también para el planteamiento de las posibles soluciones, surge de los estudios cualitativos de conocimientos, actitudes y prácticas (CAP) llevados a cabo en tres ciudades del Perú por el Programa de Investigación en Accidentes de Tránsito (PIAT) ${ }^{(15,16)}$ y cuyos resúmenes están disponibles en la página Web del Instituto Nacional de Salud ${ }^{(17)}$. En el estudio de CAP de padres y sus hijos, se encontró que los padres tenían, en general, buenos perfiles de CAP pero que necesitaban ser reforzados en los niños. Además, para muchos de los CAP evaluados, no es posible afirmar que los perfiles de CAP negativos se debían a falta de conocimiento de las reglas. Por el contrario, la gente suele conocer que está haciendo algo mal, como por ejemplo no hacer uso de los puentes peatonales y cruzar la pista en lugares no indicados ${ }^{(15)}$. El estudio CAP en choferes, peatones y policías (16) refuerza estos hallazgos al encontrar que el desconocimiento no es la principal causa del incumplimiento de las normas y la racionalidad costobeneficio prima en la comunidad cuando se decide actuar de acuerdo con una práctica que no respeta las normas ${ }^{(16)}$. De estos estudios se desprende que no es necesariamente la falta de conocimiento de las normas lo que explique el poco respeto a ellas. Se desprende también que aquellas iniciativas orientadas únicamente a hacer conocer las normas, en su esquema tradicional 
de campañas de educación, tendrán un impacto limitado, pues se debería poner mayor atención en la reducción de las relaciones de conflicto y desconfianza entre actores y en la percepción de corrupción existente de forma tan diseminada en nuestro medio.

Esto resulta fundamental, dado que una percepción subjetiva sugiere que la mayoría de traumatismos causados por el tránsito ocurren mayormente en las carreteras, un mito fuertemente alimentado por las noticias locales. Sin embargo, el Perú es el país que tiene los mayores índices de peatones afectados por este problema ${ }^{(2)}$, tal y como señala en su artículo Quistberg (14).

Por otra parte, parece legítimo preguntarse qué pasa en nuestro país, que tiene leyes relacionadas con la seguridad vial con un potencial claro para reducir el problema de los traumatismos causados por el tránsito, como aquellas que establecen penas para los conductores que conduzcan bajo los efectos del alcohol, o que excedan los límites de velocidad permitidos. Sin embargo, es claro que el grado de cumplimiento de esas leyes es muy limitado. Nuevamente, no pareciera que se trate simplemente de un problema de falta de información sobre medidas adecuadas de seguridad vial. Es preocupante la percepción que las personas tienen sobre la gobernabilidad y la rendición de cuentas. Sin duda es fundamental, para posibilitar que en el país se imponga una cultura de respeto a las leyes, a los demás y a las autoridades, el desarrollo de confianza en las instituciones y en las personas. Estas son áreas de trabajo en las que disciplinas como la antropología, la sociología y la psicología pueden contribuir sustancialmente.

\section{PROGRESOS DESDE EL SECTOR SALUD EN TRAUMATISMOS CAUSADOS POR EL TRÁNSITO EN EL PERÚ}

El panorama indicado no significa que no se haya hecho esfuerzos para enfrentar el problema. El Ministerio de Salud ha establecido la Estrategia Sanitaria Nacional en Accidentes de Tránsito (ESNAT). No son muchas las estrategias sanitarias y esta designación resulta un indicativo del nivel de prioridad que se adjudica al tema dentro del sector. La ESNAT se encuentra bajo la responsabilidad de la Oficina General de Defensa Nacional -órgano asesor del Ministerio de Salud- quien tiene a su cargo su implementación, coordinación y ejecución ${ }^{(18)}$. En este número especial la ESNAT esboza el perfil epidemiológico del problema en los últimos años ${ }^{(9)}$.

Por el lado del monitoreo, y reconociendo las limitaciones presentes con la calidad de la información en el tema, la Dirección General de Epidemiología ha establecido un sistema de información que permitiría la recopilación de aquellas víctimas atendidas por un traumatismo vinculado con colisiones. Este sistema depende de las contribuciones de las secciones de epidemiología de los establecimientos de salud del Ministerio de Salud y la información es canalizada a nivel central ${ }^{(19)}$, que hoy se publica en esta edición especial ${ }^{(10)}$.

Y, en lo que a investigación se refiere, el Instituto Nacional de Salud marcó un hito sin precedentes en la salud pública peruana al reconocer a los traumatismos causados por el tránsito como un problema de salud pública ${ }^{(20)}$, y, sobre todo, al financiar el Programa de Investigación en Accidentes de Tránsito (PIAT), cuya ejecución fue encargada a la ONG Salud Sin Límites Perú a través de un concurso público. Los resúmenes de los productos de investigación de este programa se encuentran disponibles en la página Web del Instituto Nacional de Salud (17), y algunos de ellos, dada su relevancia para la audiencia local, son publicados en este número de la Revista ${ }^{(10,11)}$

\section{EL RETO DE LOS TRAUMATISMOS CAUSADOS POR EL TRÁNSITO EN EL PERÚ}

Más allá de estos hitos en el camino del progreso, quedan pendientes varios retos locales para hacer más eficiente el vínculo que las distintas instancias del sector salud deben mantener con los diferentes actores vinculados con el tema de traumatismos causados por el tránsito. Estos retos están delimitados, de alguna manera, en las contribuciones que se publican en este número de la Revista Peruana de Medicina Experimental y Salud Pública.

Primero, las limitaciones en lo concerniente a la determinación de la magnitud del problema. Reconociendo las limitaciones existentes -unidad de análisis, temporalidad, subregistro y autoreporte $(10,19,21)$ -, en este número se presentan dos aproximaciones al problema, aprovechando las estadísticas de la Policía Nacional del Perú (9) y de la III Encuesta Nacional de Consumo de Drogas en la Población General del Perú de DEVIDA ${ }^{(7)}$. Si no se conoce el problema, no es posible identificar los puntos más críticos que requieren atención inmediata. Tampoco es posible establecer pautas claras de monitoreo ni indicadores de progreso. Es claro que las estadísticas nacionales en el tema de traumatismos vinculados con el tránsito son limitadas, incompletas, no integradas y, sobre todo, no armonizadas entre los distintos actores que las recolectan ${ }^{(10,19,21)}$.

Segundo, no se conoce la epidemiología local en detalle, del problema de traumatismos causados por 
el tránsito. Reconociendo el sostenido avance del grupo de Rey de Castro en el tema de somnolencia y conductores de transporte público, sistematizados en este número ${ }^{(6,8,22)}$, poco se ha logrado avanzar. Se podría afirmar que la mejor información de los perfiles y daños ocasionados por los traumatismos causados por el tránsito es propiedad de las empresas aseguradoras (19). No se conoce con exactitud la cantidad de muertos vinculados con los traumatismos causados por el tránsito ${ }^{(21)}$ y las noticias de primeras páginas en los diarios capturan mucho la atención del problema. Esta información sugeriría que la mayor cantidad de muertes ocurren en los vehículos de transporte interprovinciales, pero esto no es necesariamente correcto. Más aun, esta percepción -simplista dada la falta de una única fuente de información sólida- oscurece y deja de lado el problema de los traumatismos causados por el tránsito en el grupo de peatones ${ }^{(19)}$, el que ciertamente acarrea un importante impacto socioeconómico negativo ${ }^{(23,24)}$. Además, según los organismos técnicos internacionales, la definición de mortalidad vinculada con un accidente de tránsito, debe involucrar a las muertes que ocurren dentro de los 30 días que ocurre el evento ${ }^{(2,25)}$. A la fecha, esto no es posible conocerlo en detalle en nuestro medio, peor aun, la morbilidad asociada con traumatismos causados por el tránsito, medidos en términos de complicaciones resultantes y discapacidad postevento, no es conocida en detalle ${ }^{(24)}$.

Tercero, en términos de políticas públicas, se vienen implementando distintas iniciativas. Sin embargo, estas implementaciones carecen de un sólido componente de supervisión del progreso y evaluación de impacto. EI SOAT ha establecido un mecanismo de reducción de brechas para acceder a la atención, que aún tiene muchas limitantes en su parte operativa ${ }^{(11)}$. Sin embargo, como bien reza el dicho, "hecha la ley hecha la trampa"; en este caso, "hecho el SOAT, hecho el AFOCAT" (Asociaciones de Fondos Regionales o Provinciales contra Accidentes de Tránsito) (26). Como entidad neutral del Estado, la Defensoría del Pueblo del Perú, a través de la Adjuntía del Medio Ambiente, Servicios Públicos y Pueblos Indígenas, ha ejercido un liderazgo en el ejercicio de identificar falencias y exigir mejoras en varios aspectos vinculados con los traumatismos causados por el tránsito ${ }^{(27-29)}$, tal y como se detalla en el simposio de este número especial (12). Tolerancia cero, otro ejemplo de acción voceada por su puesta en marcha, no está libre de limitaciones, tal y como han sido identificadas por grupos independientes ${ }^{(29,30)}$. En su diseño se contempló a los vehículos como unidad de interés y no se establecieron indicadores de impacto en el número de afectados. En tal sentido, se espera que tenga un efecto, pero ello es difícil de cuantificar y separar ${ }^{(30)}$.
De lo anterior se desprende que la planificación, la implementación y la evaluación de intervenciones para la reducción del problema requiere una aproximación amplia, que incluye al sector salud, pero requiere igualmente el concurso de otros sectores y otras áreas.

La agenda de investigación es igualmente amplia y compleja. Si bien es importante que cada sector establezca sus propias prioridades de investigación en relación con los traumatismos causados por el tránsito, es crucial que se desarrolle una agenda inclusiva de investigación que considere los diversos aspectos del problema, tanto en el nivel nacional como en el local.

\section{AGRADECIMIENTOS}

Al Dr. Edmundo Rosales por su apoyo editorial en la coordinación de este número especial y a todos los miembros del equipo del PIAT y Salud Sin Límites Perú por su apoyo constante durante la ejecución del programa.

\section{Conflictos de Interés}

Los autores, durante el año 2009, se desempeñaron como Investigador Principal (JJM) y Coinvestigador Principal (LH) del Programa de Investigacion en Accidentes de Tránsito (PIAT) financiado por el Instituto Nacional de Salud. La institución financiera no tuvo participación en el diseño de los estudios, recolección de datos, análisis e interpretación de los resultados, ni en la redacción de los informes técnicos y artículos científicos resultantes del PIAT.

\section{REFERENCIAS BIBLIOGRÁFICAS}

1. Peden MM, Scurfield R, Sleet D, Mohan D, Hyder AA, Jarawan E, et al. World report on road traffic injury prevention. Geneva: World Health Organization; 2004.

2. World Health Organization. Global status report on road safety. Geneva: WHO; 2009.

3. Rey de Castro J, Rosales-Mayor E. Monitoreo del sueño en conductores de ómnibus y camiones: factor relevante a considerar para la renovación de la licencia de conducir. Rev Peru Med Exp Salud Publica. 2010; 27(2): 260-66.

4. Morales-Soto N, Alfaro-Basso D, Gálvez-Rivero W. Aspectos psicosociales y accidentes en el transporte terrestre. Rev Peru Med Exp Salud Publica. 2010; 27(2): 267-72.

5. Malaga $\mathbf{H}$. Medidas y estrategias para la prevención y control de los accidentes de tránsito: experiencia peruana por niveles de prevención. Rev Peru Med Exp Salud Publica. 2010; 27(2): 231-36.

6. Rey de Castro J, Rosales-Mayor E. Cansancio y somnolencia durante el desempeño laboral de los conductores interprovinciales: experiencia peruana y planteamiento de propuestas. Rev Peru Med Exp Salud Publica. 2010; 27(2): 237-42. 
7. Wong P, Gutiérrez C, Romaní F. Autorreporte de accidentes de tránsito en una encuesta nacional en la población urbana del Perú. Rev Peru Med Exp Salud Publica. 2010; 27(2): 170-78.

8. Liendo GR, Castro CL, Rey de Castro J. Cansancio y somnolencia en conductores de ómnibus interprovinciales: estudio comparativo entre formalidad e informalidad. Rev Peru Med Exp Salud Publica. 2010; 27(2): 187-94.

9. Choquehuanca-Vilca V, Cárdenas-García F, CollazosCarhuay J, Mendoza-Valladolid W. Perfil epidemiológico de los accidentes de tránsito en el Perú, 2005-2009. Rev Peru Med Exp Salud Publica. 2010; 27(2): 162-69.

10. Miranda JJ, Paca-Palao A, Najarro L, Rosales-Mayor E, Luna D, Lopez L, et al. Diagnóstico situacional, estructura, dinámica y monitoreo de los sistemas de información en accidentes de tránsito en el Perú - 2009. Rev Peru Med Exp Salud Publica. 2010; 27(2): 273-87.

11. Miranda JJ, Rosales-Mayor E, Gianella C, Paca-Palao A, Luna D, Lopez L, et al. Cobertura formal y real de la Ley de Atención de Emergencia y del Seguro Obligatorio contra Accidentes de Tránsito (SOAT). Rev Peru Med Exp Salud Publica. 2010; 27(2): 179-86.

12. Sagástegui F. Supervisando la seguridad vial en el Perú. Rev Peru Med Exp Salud Publica. 2010; 27(2):

13. Huiang CM, Lunnen JC, Miranda JJ, Hyder AA. Traumatismos causado por el tránsito en países en desarrollo: agenda de investigación y de acción. Rev Peru Med Exp Salud Publica. 2010; 27(2): 243-47.

14. Quistberg DA, Miranda JJ, Ebel B. Reduciendo el trauma y la mortalidad asociada a los accidentes de tránsito en los peatones en el Perú: intervenciones que pueden funcionar. Rev Peru Med Exp Salud Publica. 2010; 27(2): 248-54.

15. Huicho L, Miranda JJ, Luna D, López L, Paca A, Rosales $E$, et al. Estudio CAP, nivel de sensibilización, movilización, participación y fortalecimiento de organizaciones comunitarias en la prevención de daños y riesgos relacionados a accidentes de tránsito / Encuesta en padres e hijos [Informe Técnico]. Lima: Instituto Nacional de Salud, Salud sin Límites Perú; 2009.

16. Huicho L, Miranda JJ, Luna D, Paca A, López L, Rosales E, et al. Estudio CAP, nivel de sensibilización, movilización, participación y fortalecimiento de organizaciones comunitarias en la prevención de daños y riesgos relacionados a accidentes de tránsito / Estudio cualitativo, grupos focales [Informe Técnico]. Lima: Instituto Nacional de Salud, Salud Sin Límites Perú; 2009.

17. Instituto Nacional de Salud. Programa de Investigacion en Accidentes de Tránsito [Página en Internet]. Lima: INS; 2009. [Fecha de acceso: 1 de junio de 2010]; Disponible en: http://www.ins.gob.pe/insVirtual/ins/ investigacionEnSalud/InvestigacionesFinanciadasINS/ programasInvestigacionAccidentesTransito.asp.

18. Perú, Ministerio de Salud. Plan Nacional de la Estrategia Sanitaria Nacional de Accidentes de Tránsito 2009-2012. Lima: MINSA; 2009.

19. Miranda JJ, Huicho L, Paca A, Najarro L, Luna D, López L, et al. Diagnóstico situacional de los sistemas de información en accidentes de tránsito en el Perú [Informe Técnico]. Lima: Instituto Nacional de Salud, Salud sin Límites Perú; 2009.

20. Instituto Nacional de Salud. Prioridades de investigación en salud en el Perú: análisis del proceso. Lima: INS; 2007.

21. Miranda JJ, Huicho L, López L, Paca A, Rosales E, Luna D, et al. Incidencia, tendencia de los accidentes de tránsito en el Perú y factores de riesgo dependientes del peatón, vehículo y conductor [Informe Técnico]. Lima: Instituto Nacional de Salud, Salud Sin Límites Perú; 2009.

22. Rosales E, Egoavil M, Durand I, Montes N, Flores R, Rivera S, et al. Accidentes de carretera y su relación con cansancio y somnolencia en conductores de ómnibus. Rev Med Hered. 2009; 20(2): 136-47.

23. Best P, Miranda JJ, Huicho L, Paca A, Luna D, Luis L, et al. Impacto socio económico de los accidentes de tránsito [Informe Técnico]. Lima: Instituto Nacional de Salud, Salud Sin Límites Perú; 2009.

24. Best P, Miranda JJ, Huicho L, Paca A, Luna D, Luis L, et al. Complicaciones resultantes en la calidad de vida relacionada a la salud en víctimas de accidentes de tránsito [Informe Técnico]. Lima: Instituto Nacional de Salud, Salud Sin Límites Perú; 2009.

25. Jacobs G, Thomas AA, Astrop A. Estimating global road fatalities (TRL Report 445). Crowthorne: Transport Research Laboratory; 2000.

26. Defensoría del Pueblo. Supervisión a las AFOCAT de las regiones de La Liberta, Junín y Lima. Informe de la Adjuntía Nº31-2009-DP/AMASPPI-SP. Lima: Defensoría del Pueblo; 2009.

27. Defensoría del Pueblo. Informe Defensorial $N^{\circ} 108$. Pasajeros en riesgo: la seguridad en el transporte interprovincial. Lima: Defensoría del Pueblo; 2006.

28. Defensoría del Pueblo. Informe Defensorial № 137: El transporte urbano en Lima Metropolitana: Un desafío en defensa de la vida. Lima: Defensoría del Pueblo; 2008.

29. Defensoría del Pueblo. Resultados de la supervisión del Plan Tolerancia Cero en las regiones de Arequipa, Lima, Lambayeque, Tacna y Puno. Informe de Adjuntía N ${ }^{\circ} 0012-$ 2008-DP/ASPMA.SP. Lima: Defensoría del Pueblo; 2008.

30. Best P, Miranda JJ, Huicho L, Paca A, Luis L, Rosales E, et al. Evaluación de costo-efectividad de una intervención que tenga impacto en la reversión del daño: Plan Tolerancia Cero [Informe Técnico]. Lima: Instituto Nacional de Salud, Salud Sin Límites Perú; 2009.

Correspondencia: J. Jaime Miranda, MD, MSc, PhD

Dirección: Programa de Investigación en Accidentes de Tránsito (PIAT), Salud Sin Límites Perú, Calle Ugarte y Moscoso \# 450 Of. 601, Magdalena, Lima 17, Perú.

Teléfono: (511) 2615684

Correo electrónico: jaimemiranda@saludsinlimitesperu.org.pe; jaime.miranda@upch.pe 